\title{
Q-ball Formation through Affleck-Dine Mechanism
}

\author{
S. Kasuya ${ }^{1}$ and M. Kawasaki ${ }^{2}$ \\ ${ }^{1}$ Department of Physics, Ochanomizu University, Bunkyo-ku, Tokyo 112-8610, Japan \\ 2 Research Center for the Early Universe, University of Tokyo, Bunkyo-ku, Tokyo 113-0033, Japan
}

(September 25, 1999)

\begin{abstract}
We present the full nonlinear calculation of the formation of a Q-ball through the Affleck-Dine (AD) mechanism by numerical simulations. It is shown that large Q-balls are actually produced by the fragmentation of the condensate of a scalar field whose potential is very flat. We find that the typical size of a Q-ball is determined by the most developed mode of linearized fluctuations, and almost all the initial charges which the AD condensate carries are absorbed into the formed Q-balls, whose sizes and the charges depend only on the initial charge densities.
\end{abstract}

PACS numbers: 98.80.Cq, 11.27.+d, 11.30.Fs

hep-ph/9909509

A Q-ball is a non-topological soliton with some conserved global charge in the scalar field theory [1]. A Qball solution exists if the energy minimum develops at non-zero $\phi$ with fixed charge $Q$. In terms of the effective potential of the field $\phi, V(\phi) / \phi^{2}$ takes the minimum at $\phi \neq 0$ [1.2]. The Q-ball naturally appears in the spectrum of the minimal supersymmetric standard model (MSSM) [3]. In particular, very large Q-balls could exist in the theory with a very flat potential [4], such as in the MSSM which has many flat directions, which consist of squarks and sleptons, carrying baryonic and/or leptonic charges [5]. It provides interesting attention to phenomenology and astrophysics [4, 6, 6 .

Cosmologically, it is interesting that large Q-balls carrying baryonic charge (B-ball) can be promising candidates for the dark matter of the universe, and/or the source for the baryogenesis [8 10]. Moreover, they can explain why the energy density of baryons is as large as that of the dark matter (at least within a few orders of magnitude). If the effective potential of the field $\phi$ carrying the baryonic charge is very flat at large $\phi$, as in the theory that the supersymmetry (SUSY) breaking occurs at low energy scales (gauge-mediated SUSY breaking), B-ball energy per unit charge decreases as the charge increases [ [ $\left[\right.$. For large enough charge, such as $B \gtrsim 10^{12}$, the B-ball cannot decay into nucleon, and is completely stable, which implies that B-balls themselves can be the dark matter [8] with charges $B=10^{14}-10^{26}$ [11], while baryons are created by the conventional Affleck-Dine (AD) mechanism. In the case of gravity-mediated SUSY breaking scenario, the B-balls can decay into quarks or nucleons, with the decay (evaporation) rate of the Qball proportional to the surface area [12], and if they decay after the electroweak phase transition, there are some advantages over the conventional $\mathrm{AD}$ baryogenesis [9,10]. For example, B-balls can protect the baryon asymmetry from the effects of lepton violating interactions above the electroweak scale when anomalous $B+L$ violation is in thermal equilibrium. Another one is that Q-balls with $B-L$ charge survive the sphaleron effects to create the same amounts of baryon and lepton num- bers. In either case, it is necessary for Q-balls to have large charges, such as $Q=10^{22}-10^{28}$ [9,10]. In this scenario, dark matter is lightest supersymmetric particle (LSP) which arises from the Q-ball decay, and parameters of MSSM could be constrained by investigating the Q-ball cosmology [13]. Note that it is also possible to have stable Q-balls in the gravity-mediated SUSY breaking theory depending on the details of the features of the hidden sector 114.

Those large Q-balls are expected to be created through $\mathrm{AD}$ mechanism [15] in the inflationary universe [8 10]. The coherent state of the AD scalar field which consists of some flat direction in MSSM becomes unstable and instabilities develop. These fluctuations grow large to form Q-balls.

The formation of large Q-balls has been studied analytically only with linear theory $[8,10]$ and numerical simulations were done in one dimensional lattices [8]. Both of them are based on the assumption that the Q-ball configuration is spherical so that we cannot really tell that the Q-ball configuration is actually accomplished. Recently, some aspects of the dynamics of AD scalar and Q-ball formation were studied in Ref. [16], but the whole evolution was not investigated, which is important for the investigation of the Q-ball formation. (In the context of non-relativistic Bose gas, the dynamics of drops of Bose-Einstein condensate, which is a non-topological soliton, are studied in Ref. [17].) In this Rapid Communication, we study the dynamics of a complex scalar field with very flat potential numerically in one, two, and three dimensional lattices, without assuming spherical Q-ball configuration. On one dimensional lattices, it is equivalent to the system independent of other two dimensions, so that we are observing plane-like objects. We call them $Q$-walls. Likewise, string-like objects, which we call $Q$ strings, appear on two dimensional lattices.

First we show where the instabilities of a scalar field come from. To be concrete, let us assume that the complex scalar field $\Phi$ has an effective potential of the form [8] 


$$
V(\Phi)=m^{4} \ln \left(1+\frac{|\Phi|^{2}}{m^{2}}\right)-c H^{2}|\Phi|^{2}+\frac{\lambda^{2}}{M^{2}}|\Phi|^{6},
$$

where $m$ is the mass of the field, $H$ is the Hubble parameter, $\lambda$ is a dimensionless coupling constant, $M \simeq$ $2.4 \times 10^{18} \mathrm{GeV}$ is the (reduced) Planck mass, and $c$ is a constant. Hereafter, we assume the matter-dominated universe, where $H=2 / 3 t$. This form of the potential arises naturally in the gauge-mediated SUSY breaking scenario in MSSM [8]. In addition to Eq.(11), the A-term (e.g., $\left.A\left(\Phi^{4}+\Phi^{* 4}\right)\right)$ is necessary for baryogenesis, since it makes the $\mathrm{AD}$ field rotate to create baryon numbers. Here we assume that the A-term does not crucially affect other terms of the potential, and take ad hoc initial conditions neglecting the A-term. The $\mathrm{AD}$ field thus has the initial charge density. Notice that the effective potential for the flat direction has very similar form in the gravitymediated SUSY breaking scenario [9], and the features of the Q-ball formation are expected to be the same.

If we note that the field is dominated by the logarithmic potential and the homogeneous mode has only a real part (i.e., no rotational motion), neglecting the second and third term in Eq.(1), the instability band is approximately estimated as [18]

$$
\frac{k}{a} \lesssim \frac{m^{2}}{|\Phi|}
$$

for large field value $|\Phi|$, which is exactly the same as the result of Ref. [8] (note that there are additional instability bands which come from the parametric resonance effects because of the oscillation of the homogeneous field, but they are subdominant effects). Therefore, the instability band grows as time goes on, since the amplitude of the homogeneous mode $|\Phi|$ decreases as $a^{-3}$ for the logarithmic potential. These fluctuations originate from the negative curvature of the logarithmic potential, which produces negative pressure [9]. It is expected that Q-balls with corresponding scales are formed.

As we mentioned, we calculate the dynamics of the complex scalar field on one, two, and three dimensional lattices. We formulate the equation of motion for the real and imaginary part of the field: $\Phi=\frac{1}{\sqrt{2}}\left(\phi_{1}+i \phi_{2}\right)$. Rescaling variables with respect to $m$, we have dimensionless variables

$$
\varphi=\frac{\phi}{m}, \quad h=\frac{H}{m}, \quad \tau=m t, \quad \xi=m x .
$$

We have exhaustedly calculated the dynamics of the scalar field and Q-balls for various parameters, and find that Q-balls are actually formed through the AffleckDine mechanism in three dimensional lattices. They have thick-wall profiles which are approximately spherical, and its charge is conserved as time goes on. Here we show one example. Figure 1 shows the configuration of the Q-ball at $\tau=10^{6}$ on $64^{3}$ three dimensional lattices with $\Delta \xi=1.0$ in the matter dominated universe. Initial conditions are $\varphi_{1}(0)=2.5 \times 10^{6}, \varphi_{1}^{\prime}(0)=0, \varphi_{2}(0)=$

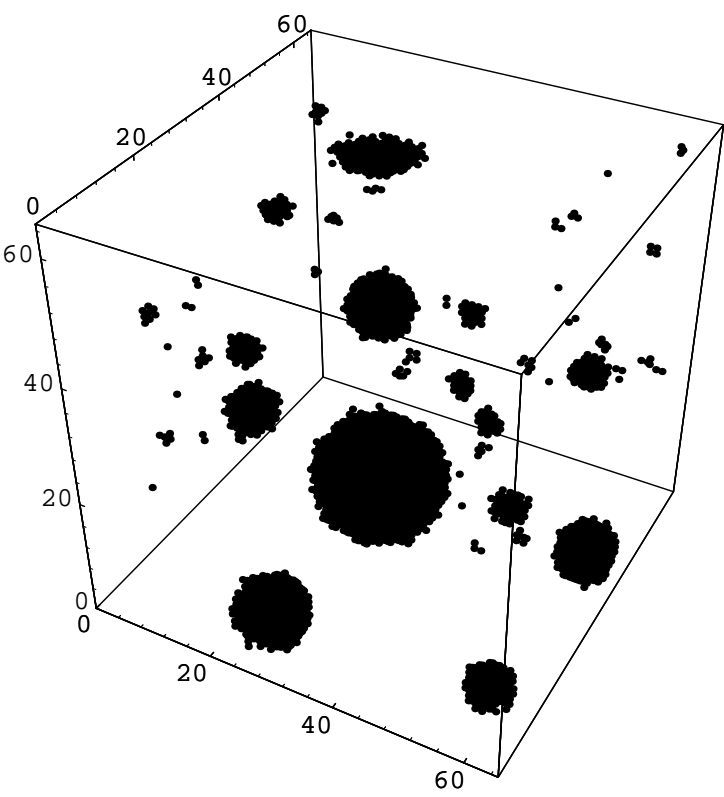

FIG. 1. Configuration of Q-balls on three dimensional lattice. More than 30 Q-balls are formed, and the largest one has the charge with $Q \simeq 1.96 \times 10^{16}$

$0, \varphi_{2}^{\prime}(0)=4.0 \times 10^{4}, \tau(0)=100$. We can see more than 30 Q-balls in the box. The charge of the largest Q-ball is $Q \simeq 1.96 \times 10^{16}$ evaluated by

$$
Q=\int d^{3} \xi q=\int d^{3} \xi \frac{1}{2}\left(\varphi_{1} \varphi_{2}^{\prime}-\varphi_{2} \varphi_{1}^{\prime}\right)
$$

Though the best way to investigate the nature of Q-ball formation is to calculate in three dimensions, we must use the large-sized box to take into account low momenta effects and the large number of lattices in order to have the enough resolution. Therefore, we have calculated in one and two dimensions, and use these results complementarily with results in three dimensions.

Therefore, let us now compare the evolutions of Q-balls in one, two, and three dimensions. As is mentioned, a Qball is a non-trivial configuration of the scalar field, which we can obtain by estimating the energy minimum with finite charge $Q$ fixed. From Eq.(四) we can approximately estimate the charge of a Q-ball as

$$
Q=a^{3} Q_{D} \sim a^{3} R^{D} \tilde{q} \sim \text { const. }
$$

where $\tilde{q}=\phi_{1} \dot{\phi}_{2}-\dot{\phi}_{1} \phi_{2}$ is the charge density, and $Q_{D}$ is the charge in $D$ dimension. Charge conservation tells us that $Q$ is constant. If we assume the form of a Q-ball as

$$
\phi(\mathbf{x}, t)=\phi(\mathbf{x}) \exp (i \omega t),
$$

the energy of a Q-ball can be calculated as

$$
\begin{aligned}
E & =\int d^{3} x\left[\frac{1}{2}(\nabla \phi)^{2}+V(\phi)-\frac{1}{2} \omega^{2} \phi^{2}\right]+\omega Q \\
& =\int d^{3} x\left[E_{\text {grad }}+V_{1}+V_{2}\right]+\omega Q,
\end{aligned}
$$


where

$$
\begin{aligned}
E_{\text {grad }} & \sim \frac{\phi^{2}}{a^{2} R^{2}}, \\
V_{1} & \sim m^{4} \log \left(1+\frac{\phi^{2}}{2 m^{2}}\right) \sim \text { const. } \\
V_{2} & \sim \omega^{2} \phi^{2} .
\end{aligned}
$$

When the energy takes the minimum value, the equipartition is achieved: $E_{\text {grad }} \sim V_{1}$ and $E_{\text {grad }} \sim V_{2}$. From these equations and the charge conservation, we obtain the following evolutions:

$$
\begin{aligned}
& R \propto a^{-4 /(D+1)}, \\
& \phi \propto a^{(D-3) /(D+1)}, \\
& \omega \propto a^{-(D-3) /(D+1)},
\end{aligned}
$$

which we observed have approximately the same features numerically [18]. Therefore, the physical size $R_{\text {phys }}=R a$ of the Q-balls for $D=1,2$ shrinks, while remaining constant for $D=3$. Thus, stable Q-balls (three dimensional objects) can be formed, but Q-walls or Q-strings shrink because of the charge conservation.

Figure 2 shows the power spectra of the cases of one dimensional lattices with the box size $L=4096$ and linearized fluctuations at $\tau=4.5 \times 10^{5}$ and $5 \times 10^{5}$. As expected, both spectra are very similar at $\tau=4.5 \times 10^{5}$, since fluctuations have not fully developed yet. After they are fully developed $\left(\tau=5 \times 10^{5}\right)$, the spectrum becomes smooth and broad because of rescattering [19] (panel 2(c)). But, even at this time, the most developed mode is the same as that of linearized fluctuations (Compare panels 2(c) and 2(d)). Therefore, we conclude that the size of Q-balls is determined by the most developed mode of linearized fluctuations when the amplitude of fluctuations becomes as large as that of the homogeneous mode, $\left\langle\delta \phi^{2}\right\rangle \sim \phi^{2}$. For the case of Fig. 2, the typical size is $k_{\max } \sim 0.04$, which implies that $R_{\text {phys }} \sim a\left(\tau_{f}\right) / k_{\max } \sim 7.3 \times 10^{3}$, where $\tau_{f}=5 \times 10^{5}$ is the Q-ball formation time. At this time, the ratio to the horizon size is $\sim 10^{-2}$, which corresponds to the results of Ref. [8], but this value may not have important meaning, since the sizes of the horizon and Q-balls have different time evolutions. This size is consistent with the actual sizes appearing on three dimensional lattices, where the largest Q-ball has the size $R_{\text {phys }} \approx 1.1 \times 10^{4}$, and the average size of the second to fifth largest Q-balls is $\simeq 5.2 \times 10^{3}$.

We also observed that almost all the initial charges carried by the condensate of $\mathrm{AD}$ field are absorbed into Qballs formed from the fragmentations of the condensate, and the amplitude of the homogeneous mode is highly damped, which means that they carry only a small fraction of the total initial charges. In the case of Fig. 1, more than $95 \%$ of the charges are stored in the Q-balls.

Actually, the charges and sizes of Q-balls depend on the initial value of the charge carried by the $\mathrm{AD}$ condensate. Since the initial charge density of AD scalar is

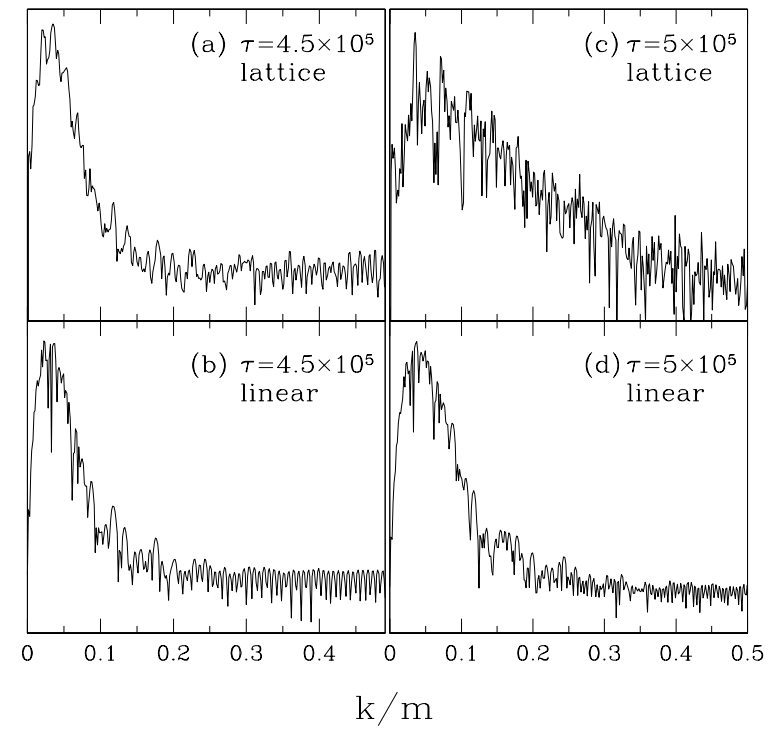

FIG. 2. Power spectrum of fluctuations of AD scalar field when the amplitude of fluctuations becomes as large as that of the homogeneous mode: $\left\langle\delta \phi^{2}\right\rangle \sim \phi^{2}$. The top panels (a) and (c) show the full fluctuations calculated on one dimensional lattices, while the bottom panels (b) and (d) show the linearized fluctuations without mode mixing.

written as $q(0)=\varphi_{1}(0) \varphi_{2}^{\prime}(0)$, the larger the initial amplitude or angular velocity of the $\mathrm{AD}$ condensate, the larger the charge stored in Q-balls. The dependences of the charge and the (comoving) size of the (largest) Qball on the initial charge density are shown in Fig. 3 . Here we take one dimensional lattices, so actually we observe Q-walls. We thus estimate their conserved charges as $Q_{\max }=a^{3} \int d x q(x)$ according to Eq.(5)). We find that one large Q-ball and a few small Q-balls are formed in most of the cases, while one Q-ball is formed in some cases such that the initial charge density $q(0)$ is relatively small. Open circles denote the dependence on $\varphi_{2}^{\prime}(0)$ for fixed $\varphi_{1}(0)$, while solid triangles denote the dependence on $\varphi_{1}(0)$ for fixed $\varphi_{2}^{\prime}(0)$ with $L=256$. Since both results show the same dependence, the only relevant variable which determines the charge and the size is the initial charge density $q(0)$. The dashed line represents the fitted line, $Q_{\max }=94.3(q(0))^{1.03}$. Crosses are obtained on $L=512$ lattices. They seem to be a little larger, and the box size effect might be remained. Notice that those Q-balls with negative charge can be produced when the initial angular velocity of the AD condensate (or, in our simulations, $\left.\varphi_{2}^{\prime}(0)\right)$ is small enough [18].

Since the (comoving) size of the Q-balls changes as time goes on, as we mentioned above, the actual values of the size at $\tau=5 \times 10^{7}$ in one dimension is not so important. What is important is how the size depends on $q(0)$. The dotted line shows the fitted line written as $R=9.46 \times 10^{-7}(q(0))^{0.464}$, though we expect the relation $R \propto(q(0))^{1 / 3}$, which means that the charge is propor- 


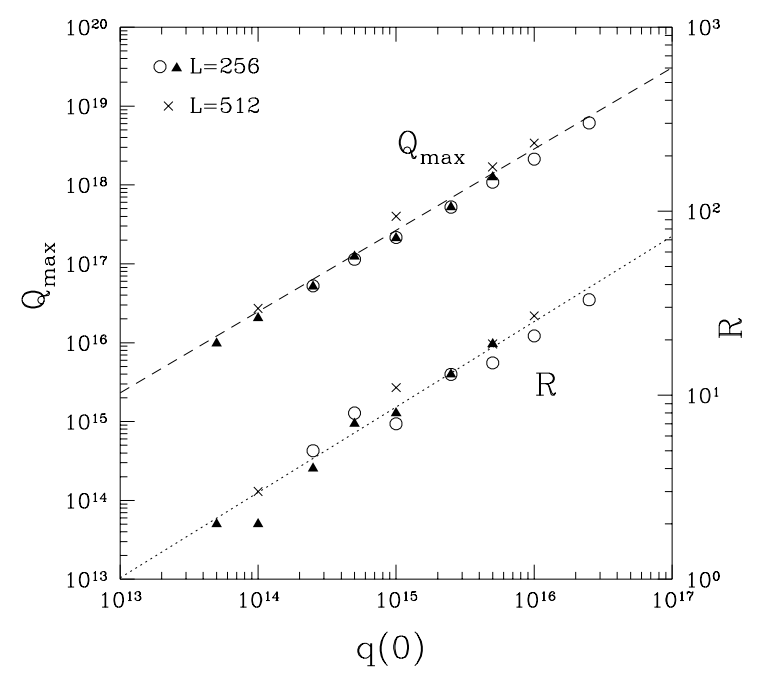

FIG. 3. Dependence of charges and sizes on the initial charge $q(0)=\varphi_{1}(0) \varphi_{2}^{\prime}(0)$ carried by the $\mathrm{AD}$ condensate on one dimensional lattices. Open circles and solid triangles denote the dependence on $\varphi_{2}^{\prime}(0)$ and $\varphi_{1}(0)$, respectively. Crosses are obtained on larger box size. $R$ is evaluated at $\tau=5 \times 10^{7}$. Note that the relevant variable is only $q(0)$, since both dependences lie on the same lines.

tional to the volume as in Eq.(5). It may be one of the reasons for the discrepancy that the values of $R$ for small charge Q-balls may have considerable error because of poor resolution in spatial lattices.

In conclusion, we consider the full nonlinear equations of motion of the Affleck-Dine scalar field in order to see the formation of the Q-ball through the Affleck-Dine mechanism by numerical simulations. It is shown that large Q-balls are actually produced by the fragmentation of the condensate of a scalar field whose potential is very flat, as in the supersymmetric standard theory.

We find that the typical size of Q-balls is determined by that of the most developed mode of linearized fluctuations when the amplitude of fluctuations grows as large as that of the homogeneous mode: $\left\langle\delta \phi^{2}\right\rangle \sim \phi^{2}$. Almost all the initial charges carried by the $\mathrm{AD}$ condensate are absorbed into the Q-balls formed, leaving only a small fraction in the form of the remaining coherently oscillating $\mathrm{AD}$ condensate. We thus can constrain parameters of MSSM through the fraction of the baryon in Q-balls $\left(f_{B}\right)$ in the context of the Q-ball decay producing LSP dark matter 13

Moreover, the actual sizes and the charges stored within Q-balls depend on the initial charge density of the $\mathrm{AD}$ condensate, which is in good agreement with the condition of the existence of the Q-ball; that is, it exists if the scalar field can take non-trivial energy minimum configuration with a fixed charge. Therefore, Q-balls with huge charges necessary for Q-balls to be dark matter could be produced if the initial charge density that the $\mathrm{AD}$ condensate carries is large enough.

We also find that the evolution of Q-balls crucially depends on the form of their dimensions, and the stable Q-balls can only exist in the form of three dimensional objects. Smaller dimensional objects such as Q-walls and Q-strings shrink as the universe expands.

Finally, we will mention the Q-axiton (the higher energy state Q-ball), which was studied in Ref. [16]. In our simulations, Q-balls are actually formed. The field orbits in the complex plane inside Q-balls are almost complete circles. Moreover, even if the initial AD field orbit is extremely oblique such that the initial angular velocity is very small so as to create the negatively charged Qballs, circular orbits can be seen inside both positive and negative Q-balls [18]. It thus seems that Q-axitons may appear, if ever, at the very beginning of the Q-ball formation.

M.K. is supported in part by the Grant-in-Aid, Priority Area "Supersymmetry and Unified Theory of Elementary Particles"(\#707).

[1] S. Coleman, Nucl. Phys. B262, 263 (1985).

[2] A. Kusenko, Phys. Lett. B404, 285 (1997).

[3] A. Kusenko, Phys. Lett. B405, 108 (1997).

[4] G. Dvali, A. Kusenko, and M. Shaposhnikov, Phys. Lett. B417, 99 (1998).

[5] M. Dine, L. Randall, and S. Thomas, Nucl. Phys. B458, 291 (1996).

[6] A. Kusenko, M. Shaposhnikov, P.G. Tinyakov, and I.I.Tkachev, Phys. Lett. B423, 104 (1998).

[7] A. Kusenko, V. Kuzmin, M. Shaposhnikov, and P.G. Tinyakov, Phys. Rev. Lett. 80, 3185 (1998).

[8] A. Kusenko and M. Shaposhnikov, Phys. Lett. B418, 46 (1998).

[9] K. Enqvist and J. McDonald, Phys. Lett. B425, 309 (1998).

[10] K. Enqvist and J. McDonald, Nucl. Phys. B538, 321 (1999).

[11] M. Laine and M. Shaposhnikov, Nucl. Phys. B532, 376 (1998).

[12] A. Cohen, S. Coleman, H. Georgi, and A. Manohar, Nucl. Phys. B272, 301 (1986).

[13] K. Enqvist and J. McDonald, Phys. Lett. B440, 59 (1998).

[14] K. Enqvist and J. McDonald, Phys. Rev. Lett. 81, 3071 (1998).

[15] I. Affleck and M. Dine, Nucl. Phys. B249, 361 (1985).

[16] K. Enqvist and J. McDonald, hep-ph/9908316.

[17] S.Yu. Khlebnikov and I.I. Tkachev, hep-ph/9902272.

[18] S. Kasuya and M. Kawasaki, in preparation.

[19] S.Yu. Khlebnikov and I.I. Tkachev, Phys. Rev. Lett. 77, 219 (1996). 